\title{
ON THE SECULAR MOTION OF THE JOVIAN PLANETS
}

\author{
M. GHIL, F. VARADI AND W. M. KAULA \\ IGPP, University of California, Los Angeles, CA 90095-1567
}

\begin{abstract}
The motion of the Jovian planets is investigated using Hamiltonian perturbation theory and numerical integrations. Experiments varying the mass of Neptune exhibit 1:1 secular resonance between the perihelion motions of Jupiter and Uranus.
\end{abstract}

The current view of Solar system dynamics on the time-scale of millions of years is based on secular perturbation theory and various features of the dynamics are explained in terms of resonances and associated separatrices (Laskar, 1990; Sussman and Wisdom, 1992; Duncan and Quinn, 1993). The road to chaos goes through bifurcations: to understand "islands" of chaotic and regular motion in the present Solar system we need to explore the parameter space around it. We carried out numerical simulations of the motion of the Jovian planets and analyzed the results using Hamiltonian perturbation theory in order to see how well we can explain the dynamics and to find possible sources of chaos.

While developing a Hamiltonian secular perturbation theory for the Jovian planets, one has to deal with the 2:5 mean-motion near-resonance between Jupiter and Saturn, the so-called Great Inequality (GI). We found that straightforward averaging is not likely to provide a convergent perturbation expansion: the contribution from the GI at third order in the masses is comparable to its contribution at second order (Varadi et al., 1995).

To better understand the results of our numerical integrations, we derived a Lie series, retaining only 9000 terms out of about one hundred thousand, to remove all short-periodic perturbations, except for the GI. The GI prevents us at present from deriving a sufficiently accurate secular system. It does not contribute to the quadratic part of the secular Hamiltonian but it does affect higher-degree terms. The diagonalization of this quadratic Hamiltonian decouples the linear part of the secular motion for 


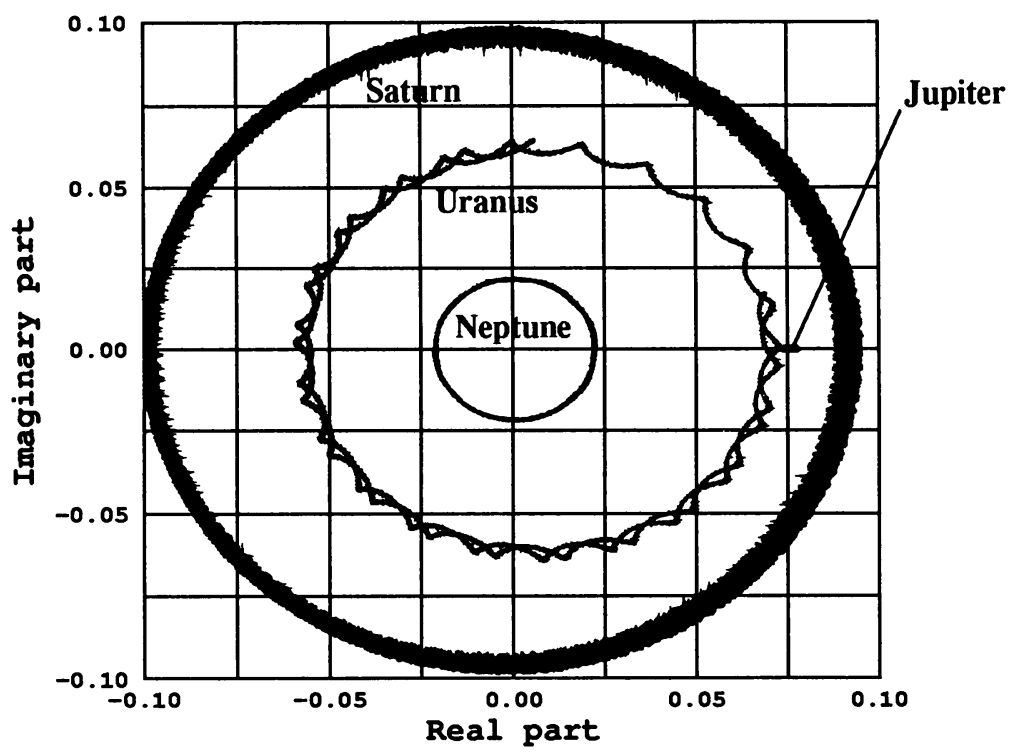

Figure 1. The diagonalized secular motion of the Jovian planets relative to Jupiter's, in $\zeta$ variables (see text for details).

each planet from that of the others, further simplifying the actual orbits. We use complexified canonical versions of the usual $h$ and $k$ variables, i.e., $\zeta=\sqrt{2 \sqrt{a}\left(1-\sqrt{1-e^{2}}\right)} \exp (-i \varpi)$, where $a$ is the semi-major axis, $e$ the eccentricity, and $\varpi$ the longitude of perihelion. The diagonalizing transformation introduces linear combinations of the original $\zeta$ variables and it is customary to associate these new variables with particular planets.

After the above transformations, the motion still exhibits large, supposedly nonlinear, variations: these are mostly dependent on the phase of the "diagonalized Jupiter's" $\zeta$. One can plot the same data relative to this phase, i.e., rotated $(h, k)$ variables, to obtain Fig. 1. Comparing this plot to a typical one for the original variables (e.g., Quinn et al., 1991), we obtain a much simpler picture. One can observe nearly circular "orbits" but the centers of these are shifted from the origin: Saturn's to the left, Uranus' and Neptune's to the right. The low- and high-variability phases of Saturn on the left and on the right of the plot, as it turns out, are caused by the GI. Furthermore, Saturn goes around the center in about 50 thousand years, disturbing the motion of Uranus and causing its oscillations in $(h, k)$.

How far is the system from a possible 1:1 secular resonance between Jupiter and Uranus? The quadratic part of the secular Hamiltonian predicts the location of secular resonances in parameter space. The simplest and least intrusive way to bring Jupiter and Uranus close to this resonance 


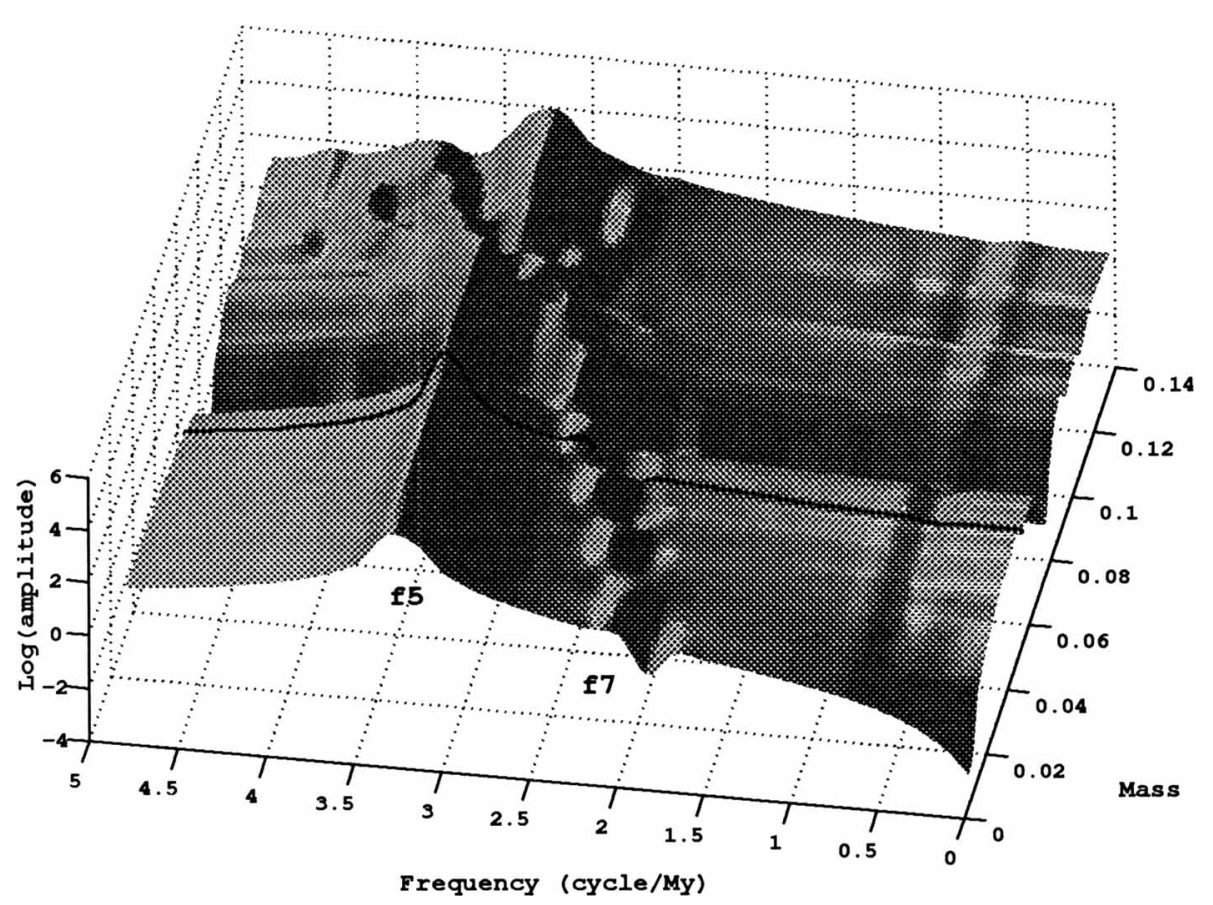

Figure 2. Composite raw Fourier spectra of Jupiter's canonical, non-diagonalized, $k=e \cos \varpi$ variable for 40 numerical integrations. The "mass" coordinate is $m_{\mathrm{N}} / m_{\mathrm{J}}$. The actual Solar System is indicated by a heavy line on the surface. The crossing by $f_{7}$ of the $f_{5}$ ridge indicates passage through the 1:1 resonance.

is to change the mass of Neptune. This does not alter the location of the mean-motion resonances nor the dynamics of the GI appreciably. Neptune's actual mass is 0.054 times that of Jupiter, $m_{\mathrm{N}}=0.054 m_{\mathrm{J}}$, and the predicted secular near-resonance is around $m_{\mathrm{N}}=0.11 m_{\mathrm{J}}$.

Near a 1:1 secular resonance, the identification of the eigenvalues and the eigenvectors of the quadratic part of the Hamiltonian breaks down. There are two nearly equal eigenvalues, and it is no longer clear how to match these to particular planets. In order to locate and understand this resonance, we carried out about 40 numerical integrations, each 6 million years long, varying $m_{\mathrm{N}}$. The symplectic mapping technique of Wisdom and Holman (1991) was used with a 100-day stepsize. Additional integrations were carried out with a 16th order Cowell-Störmer integrator developed by W. I. Newman. The case of $m_{\mathrm{N}}=0.11 m_{\mathrm{J}}$ shows large changes not only in the motion of Uranus and Neptune, but also in the motion of Jupiter. As opposed to the familiar annulus picture (e.g., Quinn et al., 1991), the plot of Jupiter's non-diagonalized $\zeta$ fills a whole disk. 
Each of the 40 integrations was analyzed separately, computing raw Fourier spectra for the non-diagonalized variables. We compiled the spectra of Jupiter's $\operatorname{Re}(\zeta)$ into a function which also depends on the mass of Neptune. This enables us to see how the main lines associated with Jupiter $\left(f_{5}\right)$ and Uranus $\left(f_{7}\right)$ behave. We obtain a surface which shows that the system does go through the 1:1 secular resonance (Fig. 2). As the mass of Neptune increases the frequency $f_{7}$ increases and, around $m_{\mathrm{N}}=0.11 m_{\mathrm{J}}$, $f_{7}$ and $f_{5}$ cannot be easily associated with individual planets. For larger Neptune masses the identity of the frequencies is clear again since $f_{5}$ 's amplitude is larger than $f_{7}$ 's. We find that the $f_{7}$ frequency is larger than $f_{5}$, i.e., the system does go through the 1:1 secular resonance.

There are some intriguing features in the composite Fourier spectra on Fig. 2. We see a large valley between $m_{\mathrm{N}}=0.06 m_{\mathrm{J}}$ and $m_{\mathrm{N}}=0.08 m_{\mathrm{J}}$ which might be a strong resonance, probably the actual 1:1 secular resonance. Also, $f_{7}$ 's ramp leading up to the main ridge of $f_{5}$ has steps which are associated with small valleys; perhaps these are other resonances. It is too early to draw definite conclusions, as the length and the accuracy of the integrations should be improved further. Also, the Wisdom-Holman mapping might have artificial bifurcations; these are being investigated by $\mathrm{W}$. I. Newman and M. Haberkorn. The main feature, however, i.e., the passage through the 1:1 resonance, was confirmed by more accurate integrations (not shown).

\section{Acknowledgements}

We thank Dr. W. I. Newman and Mr. K. R. Grazier for numerous discussions on the topic and for providing us the Cowell-Störmer integrator. The financial support of NSF Grant ATM90-13217 (MG and FV), NASA Grant NAGW-2269 (WMK \& FV) and the Condorcet Chair of the Ecole Normale Supérieure, Paris (MG) is gratefully acknowledged.

\section{References}

Duncan, M. J. and Quinn, T.: 1993, 'The long-term dynamical evolution of the Solar System', Annu. Rev. Astron. Astrophys., 31, 265

Laskar, J.: 1990, 'The chaotic motion of the Solar System: a numerical estimate of the size of the chaotic zones', Icarus, 88, 266-291

Quinn, T. Q., Tremaine, S. and Duncan, M.: 1991, 'A three million year integration of the Earth's orbit', Astron. J., 101, 2287-2305

Sussman, G. J. and Wisdom, J.: 1992, 'Chaotic evolution of the Solar System', Science, 256-257

Varadi, F., Ghil, M. and Kaula, W. M.: 1995, 'The Great Inequality in a Hamiltonian planetary theory', in From Newton to Chaos, A. E. Roy and B. A. Steves (eds.), Plenum Publ. Co., 103-108

Wisdom, J. and Holman, M.: 1991, 'Symplectic maps for the $N$-body problem', Astron. $J .$, 102, 1528-1538 